\title{
Transverse Bragg-Resonant Enhancement of Modulation and Switching
}

\author{
Wei Liang, Yong Xu, John M. Choi, Amnon Yariv, and Willie Ng
}

\begin{abstract}
We demonstrate theoretically that the electrooptic phase retardation, hence, modulation, can be enhanced very substantially in transverse Bragg resonance (TBR) waveguides. This enhancement is due fundamentally to the strong dependence of the dispersion of the TBR waveguide on the Bragg condition. Both theoretical and numerical analysis show that this enhancement can be used to improve the performance of directional coupler switches and electroabsorption modulators, leading potentially to major reductions in the device size or the switching voltage/current.
\end{abstract}

Index Terms-Electrooptic modulation, electrooptic switches, optical directional couplers, waveguides.

\section{INTRODUCTION}

$\mathbf{K}$ EY COMPONENTS in optical communication technology depend on electrically induced changes in either: 1) the index of refraction, such as electrooptic amplitude/phase modulators and directional coupler switches [1], [2] or 2) the absorption coefficient, such as in electroabsorption modulators [3]. Improvements of such devices in terms of requisite modulation voltage or length of modulator, have been achieved either by using improved materials or by using slow-wave structures [4], [5]. While the aforementioned slow-wave structure "slows" light due to the weak coupling between two neighboring cavities [6], [7], in this letter, we analyze the transverse Bragg resonance (TBR) waveguide, in which the light is slowed due to the folded optical path, as shown in Fig. 1 [8], [9]. We demonstrate theoretically that the electrooptic effect can be enhanced significantly in the TBR waveguide due to its unique dispersion characteristics [9], [10]. Particularly, we propose and analyze directional coupler switches and electroabsorption modulators based on TBR waveguides.

\section{THEORETICAL ANALYSIS}

A single TBR waveguide, as shown in Fig. 1, is composed of a guiding channel (with dielectric constant $\varepsilon_{\mathrm{co}}$ and width $W_{\text {co }}$ ) bordered by two Bragg reflectors, which alternate between a high index layer with dielectric constant $\varepsilon_{1}$ and layer thickness $L_{1}$, and a low index layer with dielectric constant $\varepsilon_{2}$ and

Manuscript received July 29, 2003; revised June 8, 2004. This paper was supported in part by Air Force Office of Scientific Research (AFOSR), in part by the Defense Advanced Research Projects Agency (DARPA), in part by the Department of Defence, and in part by the American Society of Engineering Education (ASEE).

W. Liang, Y. Xu, J. M. Choi, and A. Yariv are with the Department of Applied Physics, California Institute of Technology, Pasadena, CA 91125 USA (e-mail: liangwei@its.caltech.edu).

W. Ng is with HRL Laboratories, LLC, Malibu, CA 90265 USA

Digital Object Identifier 10.1109/LPT.2004.834559

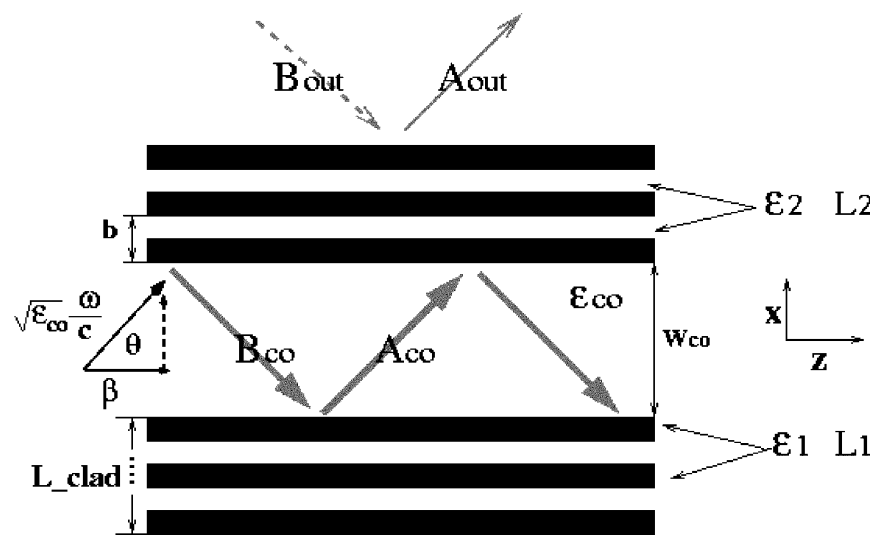

Fig. 1. Schematic of light propagation in a TBR waveguide.

layer thickness $L_{2}$. From a coupled-mode theory, it was demonstrated that low-loss guiding in TBR waveguides only occurs under the condition of TBR [9], [10]. We first consider the transverse electric (TE) mode, which can be expressed as $E_{y}(\vec{r}, t)=$ $E_{y}(x) \cdot \exp [i(\beta z-\omega t)]$ and satisfies the scalar wave equation [11]

$$
\frac{\partial^{2}}{\partial x^{2}} E_{y}(x)+k_{0}^{2} \varepsilon(x) E_{y}(x)=\beta^{2} E_{y}(x)
$$

where $k_{0}=\omega / c$ is the vacuum-wave vector, $\varepsilon(x)$ is the dielectric constant, and $\beta=\beta_{R}+i \beta_{I}$ is a complex number, where $\beta_{R}$ represents the propagation constant and $\beta_{I}$ accounts for the modal loss. Typically, $\beta_{I}$ is much smaller than $\beta_{R}$. We notice that (1) is formally the same as the one-dimensional (1-D) Schrodinger equation. As a result, for a given electrooptically induced dielectric constant change $\Delta \varepsilon(x)$, we can use the standard quantum perturbation theory to find the corresponding change of the eigenvalue $\beta^{2}$ as [12]

$$
\Delta\left(\beta^{2}\right) \approx \frac{k_{0}^{2} \int \Delta \varepsilon(x)\left|E_{y}(x)\right|^{2} d x}{\int_{-\infty}^{\infty}\left|E_{y}(x)\right|^{2} d x}=k_{0}^{2} \sum_{i} \Delta \varepsilon_{i} \Gamma_{i}
$$

where we assume $\Delta \varepsilon(x)$ takes a constant value of $\Delta \varepsilon_{i}$ within the $i$ th layer in Fig. 1 , and $\Gamma_{i}=$ $\oint_{i \text { th layer }} d x\left|E_{y}(x)\right|^{2} / \int_{-\infty}^{\infty} d x\left|E_{y}(x)\right|^{2}$ is the field confinement factor of the $i$ th layer. If we assume $\Delta \varepsilon(x)$ is the same for all the layers, i.e., $\Delta \varepsilon_{i}=\Delta \varepsilon$, then (2) can be simplified to

$$
\Delta \beta \approx \frac{k_{0}^{2} \Delta \varepsilon}{(2 \beta)}
$$

In the case of small index perturbation, i.e., $\varepsilon_{1} \approx \varepsilon_{2} \approx \varepsilon_{c o}$, a simple dispersion relation can be derived for a guided TBR 


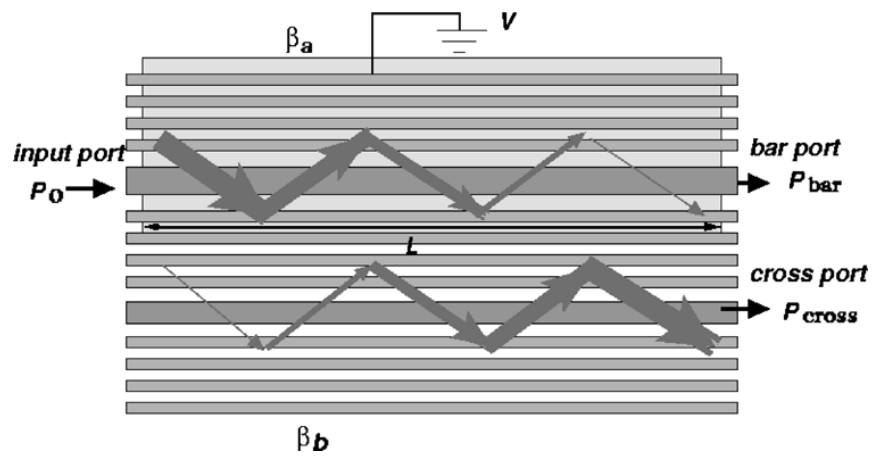

Fig. 2. Configuration of a TBR directional coupler switch.

mode $\beta=\sqrt{\bar{\varepsilon} k_{0}^{2}-(\pi / b)^{2}}[9],[10]$, where $\bar{\varepsilon}$ and $b=L_{1}+L_{2}$ are, respectively, the average dielectric constant and the periodicity of the cladding layers. Therefore, $\beta$ can be engineered by choosing $b$ and we immediately note that in (3), if we choose $\beta \ll k_{0}$, even a relatively small $\Delta \varepsilon$ can result in a large $\Delta \beta$, which effectively enhances the electrooptic effect. To characterize this enhancement, consider a conventional low-contrast slab waveguide, whose core refractive index is very close to that of the cladding material (i.e., $n=n_{\text {core }} \approx n_{\text {clad }}$ ). In this case, we have $\beta \approx n k_{0}$ and $\Delta \beta \approx k_{0} \Delta \varepsilon /(2 n)$. Thus, the electrooptic effect in the TBR waveguide is enhanced by a factor

$$
F=\frac{n k_{0}}{\beta} .
$$

Intuitively, this is because the effective optical length (or length of interaction with the material) in a TBR waveguide is increased by a factor of $n k_{0} / \beta$, as illustrated by the "zigzag" line in Fig. 1. Using these results, we consider directional coupler switches and electroabsorption modulators based on the TBR waveguide and analyze how this folded-path effect can be used to enhance the electrooptic or electroabsorption effects. In our formalism, these two cases can be treated equivalently, except that the dielectric constant change $\Delta \varepsilon$ is real in the first case and imaginary in the second one.

We first consider a TBR directional coupler switch as shown in Fig. 2. When unbiased, the propagation constants of the two arms of the TBR coupler in Fig. 2 match each other. If we choose the coupling strength between the two coupler arms $(\kappa)$ and the coupler length $(L)$, such that $\kappa L=\pi / 2$, the optical signals that enter into the input port should emerge from the cross port (see Fig. 2) [11]. Then, we apply a voltage $V_{\mathrm{DC}}$ on arm $a$ and change the real part of the dielectric constant by an amount of $\Delta \varepsilon \propto V_{\mathrm{DC}}$. If the resultant change in the propagation constant of arm $a$ satisfies $\left|\Delta \beta_{a}\right| L=\sqrt{3} \pi$, the input signal is switched from the cross port to the bar port. Although the operation principle of a TBR directional coupler switch is the same as that of a directional coupler switch based on conventional slab waveguides, with the same change of dielectric constant, the change of propagation constant of a TBR waveguide is enhanced by a factor of $F=n k_{0} / \beta$, reducing either the switching voltage or the device length by the same factor.

As for the elctroabsorption modulator based on the TBR waveguide, the operation principle is also similar to a typical electroabsorption modulator, i.e., the optical modulation is

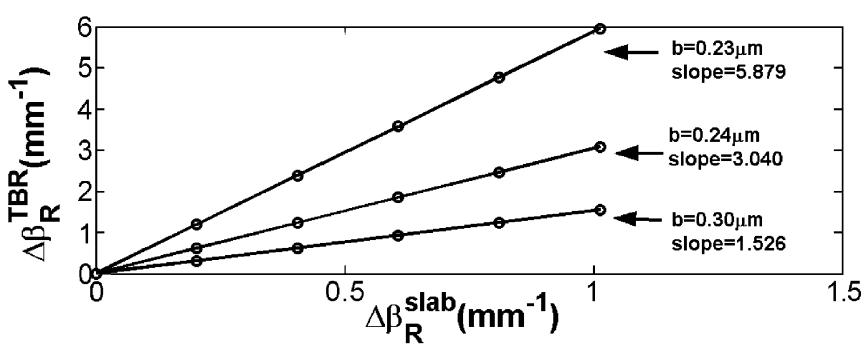

(a)

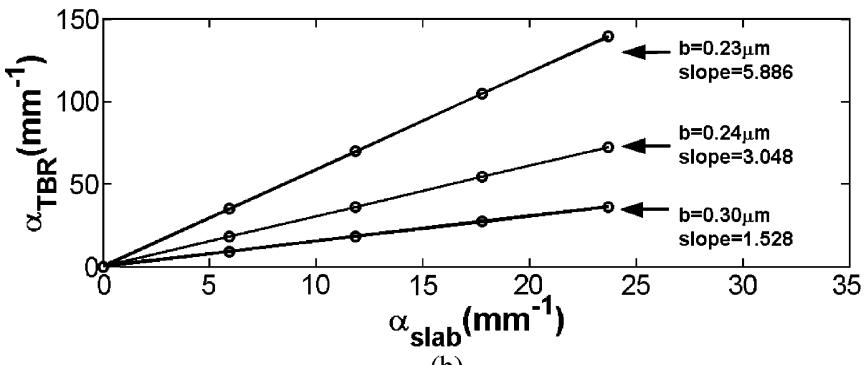

(b)

Fig. 3. (a) Relation between the electrooptically induced propagation constant change of a TBR waveguide $\Delta \beta_{R}^{\mathrm{TBR}}$ and that of a low-index contrast slab waveguide $\Delta \beta_{R}^{\text {slab }}$. (b) Relation between the electrooptically induced modal loss of a TBR waveguide $\alpha_{\mathrm{TBR}}$ and that of a low index contrast slab waveguide $\alpha_{\text {slab }}$. In both (a) and (b), the three lines are obtained using $b=0.3,0.24$, and $0.23 \mu \mathrm{m}$, respectively, which lead to different enhancement factors.

achieved by altering the absorption spectra of multiple quantum wells with external electrical signals [3]. To account for the absorption, we introduce an imaginary part $\Delta \varepsilon=i \varepsilon_{I}$ to the material dielectric constant, and use $\alpha=2 \beta_{I}$ to describe the attenuation of the optical signal. The perturbation analysis shows again that for the same external electrical signals, the signal attenuation coefficient $\alpha$ in a TBR waveguide is enhanced by a factor of $F=n k_{0} / \beta$ as compared to a conventional low-contrast slab waveguide, which means the length of the TBR device can be significantly reduced.

\section{NUMERICAL RESULTS}

In this section, we numerically calculate the complex propagation constant $\beta$ of the TE mode in a TBR waveguide and verify the above analytical predictions. The electric field in each layer of the TBR waveguide shown in Fig. 1 can be written as

$$
E_{y}(\vec{r}, t)=\left(A_{i} e^{i k_{i} x}+B_{i} e^{-i k_{i} x}\right) e^{i(\beta \cdot z-\omega t)}
$$

where $A_{i}$ and $B_{i}$ are constant coefficients in the $i$ th layer and $k_{i}=\sqrt{\left(n_{i} k_{0}\right)^{2}-\beta^{2}}$ is the transverse wavevector in the $i$ th layer. For the fundamental even TE mode, we choose the coefficients in the core area (see Fig. 1) to be normalized $A_{\text {core }}=$ $B_{\text {core }}=1$. The coefficients $A_{\text {out }}$ and $B_{\text {out }}$ at the edge of the Bragg reflector can be calculated using a transfer matrix method as described in [13]. Since a physical solution should have no incoming wave outside the Bragg reflector $\left(B_{\text {out }}=0\right)$, by minimizing $B_{\text {out }}$, we can find the complex propagation constant $\beta$ numerically.

In our calculations, we choose $\lambda=1.55 \mu \mathrm{m}, \varepsilon_{\mathrm{co}}=\varepsilon_{2}=$ $3.37^{2}, \varepsilon_{1}=3.47^{2}$ (corresponding to a GaAs-based device), $L_{1}=L_{2}=b / 2, W_{\mathrm{co}}=b$, and the number of cladding layers $N_{\text {clad }}=100$. As shown in Fig. 3, we use three values 
of $b$ for the Bragg reflector cladding, which leads to three different values of $\beta$ and enhancement factor $F$. In the case of the TBR directional coupler switch, we calculate the change of the propagation constant of the TBR waveguide $\left(\Delta \beta_{R}^{\mathrm{TBR}}\right)$ by assuming that the external electric field changes the refractive indices of both the core and the cladding by the same amount $\Delta n$. The corresponding propagation constant change in a low-index contrast slab waveguide is $\Delta \beta_{R}^{\text {Slab }}=\Delta n k_{0}$. In Fig. 3(a), we plot $\Delta \beta_{R}^{\mathrm{TBR}}$ as a function of $\Delta \beta_{R}^{\mathrm{Slab}}$, where the linear slope should give us the enhancement factor $F$. In the case of the electroabsorption modulator, we introduce a small imaginary part $\Delta \varepsilon=i \varepsilon_{I}$ into the core and cladding dielectric constants, and obtain the modal loss of the TBR waveguide $\left(\alpha_{\mathrm{TBR}}\right)$ from the imaginary part of the complex propagation constant, $\alpha_{\mathrm{TBR}}=$ $2 \beta_{I}$. For low index contrast slab waveguides, the corresponding modal loss is $\alpha_{\text {slab }}=k_{0} \varepsilon_{I} / \sqrt{\bar{\varepsilon}}$ ). The relation $\alpha_{\mathrm{TBR}} \propto \alpha_{\text {slab }}$ is plotted in Fig. 3(b), together with the linear slope, which again gives us the enhancement factor $F$. In both cases, this factor can also be calculated directly using (4) and the dispersion relation $\beta=\sqrt{\bar{\varepsilon} k_{0}^{2}-(\pi / b)^{2}}$, in which we simply use $\bar{\varepsilon}=\left(\varepsilon_{1}+\varepsilon_{2}\right) / 2$ and $\bar{n}=\sqrt{\bar{\varepsilon}}$, for the values of $b$ chosen, $0.3,0.24$, and $0.23 \mu \mathrm{m}$, we find $F$ is equal to $1.526,3.033$, and 5.824 , respectively. These results are in reasonable agreement with those obtained from the slopes shown in Fig. 3. The difference between the two approaches can be attributed to the relatively large index contrast of the TBR waveguides $(\Delta n=0.1)$.

In conclusion, we propose that TBR waveguides can be used to improve the performance of directional coupler switches and electroabsorption modulators by reducing the device length. Both analytical and numerical analysis show that the electrooptic effects can be enhanced by a factor $F=n k_{0} / \beta$ in TBR waveguides. In our design examples, this factor was found to be as large as five.

\section{REFERENCES}

[1] J. C. Campbell, F. A. Blum, D. W. Shaw, and K. L. Lawley, "GaAs electro-optic directional coupler switch," Appl. Phys. Lett., vol. 27, pp. 202-205, 1975.

[2] M. Kohtoku, K. Kawano, S. Sekine, H. Takeuchi, N. Yoshimoto, M. Wada, T. Ito, M. Yanagibashi, S. Kondo, Y. Noguchi, and M. Naganuma, "High-speed InGaAlAs-InAlAs MQW directional coupler waveguide switch modules integrated with a spotsize converter having a lateral taper, thin-film core, and ridge," J. Lightwave. Technol., vol. 18, pp. 360-369, Mar. 2000.

[3] K. Yoshino, T. Takeshita, I. Kotaka, S. Kondo, Y. Noguchi, R. Iga, and K. Wakita, "Compact and stable electroabsorption optical modulator modules," J. Lightwave Technol., vol. 17, pp. 1700-1707, Sept. 1999.

[4] N. Shaw, W. J. Stewart, J. Heaton, and D. R. Wight, "Optical slow-wave resonant modulation in electro-optic GaAs/AlGaAs modulators," Electron. Lett., vol. 35, pp. 1557-1558, Sept. 1999.

[5] M. Soljacic, S. G. Johnson, S. Fan, M. Ibanescu, E. Ippen, and J. D. Joannopoulos, "Photonic-crystal slow-light enhancement of nonlinear phase sensitivity," J. Opt. Soc. Amer. B, vol. 19, pp. 2052-2059, Sept. 2002.

[6] S. Mookherjea and A. Yariv, "Coupled resonator optical waveguides," IEEE J. Select. Topics Quantum Electron., vol. 8, pp. 446-448, June 2002.

[7] C. M. de Sterke, "Superstructure gratings in the tight-binding approximation," Phys. Rev. E, vol. 57, pp. 3502-3509, 1998.

[8] A. Y. Cho, A. Yariv, and P. Yeh, "Observation of confined propagation in Bragg waveguides," Appl. Phys. Lett., vol. 30, pp. 471-472, 1977.

[9] A. Yariv, "Coupled-wave formalism for optical waveguiding by transverse Bragg reflection," Opt. Lett., vol. 27, pp. 936-938, June 2002.

[10] J. M. Choi, W. Liang, Y. Xu, and A. Yariv, "Loss optimization of transverse Bragg resonance waveguides," J. Opt. Soc. Amer. A, vol. 21, pp. 426-429, 2004.

[11] A. Yariv, Optical Electronics in Modern Communications. Cambridge, U.K.: Oxford Univ. Press, 1997, pp. 492, 521-524.

[12] J. J. Sakurai, Modern Quantum Mechanics, revised ed. Reading, MA: Addison-Wesley, 1994, pp. 289-293.

[13] A. Yariv and P. Yeh, Optical Waves in Crystals. New York: Wiley, 1984, pp. 165-169. 